\title{
186. Studies on Human Haptoglobins. IV
}

\section{Peroxidase-like Activity and Oxygen-equilibria of the Intermediate and Saturated Forms of Hemoglobin-Haptoglobin 1-1 Complex}

\author{
By Shunjiro Kagryama, Shigeru Fujita, Akira Ogawa, \\ Kazunori KaWAMURA, and Toshiyuki YaWASE \\ Frist Department of Medicine, Faculty of Medicine, \\ Kyushu University, Fukuoka \\ (Comm. by Tanemoto Furuhata, M. J. A., Sept. 12, 1970)
}

Introduction. In the previous communications of this series, ${ }^{1,2)}$ two different hemoglobin-haptoglobin ( $\mathrm{Hb}-\mathrm{Hp})$ 1-1 complexes were demonstrated on acrylamide gel electrophoresis of $\mathrm{Hp}$ partially saturated with $\mathrm{Hb}$. From the physicochemical and immunological characterization of these complexes, the authors postulated that the intermediate and saturated forms of $\mathrm{Hb}-\mathrm{Hp} \mathrm{1-1}$ complex would be $\mathrm{Hp} 1-1$ bound to an $\alpha \beta$-subunit and two $\alpha \beta$-subunits of $\mathrm{Hb} \mathrm{A}$, respectively. ${ }^{1), 2)}$

The present communication is concerned with the peroxidase-like activity and the oxygen-equilibria of these two complexes to gain further insight into the binding mechanism of $\mathrm{Hb}-\mathrm{Hp}$ complex.

Materials and methods. Purification of $\mathrm{Hb}-\mathrm{Hp}$ 1-1 complexes. The intermediate and saturated forms were isolated from crude $\mathrm{Hp}$ 1-1 undersaturated with $\mathrm{Hb}$, and these preparations were ascertained to be homogeneous since each of them showed a single band on starch and acrylamide gel electrophoresis at alkaline $\mathrm{pH}$, and gave a single symmetrical peak on the ultracentrifugal diagram as reported previously. ${ }^{1), 2)}$

The peroxidase-like activity of Hb-Hp 1-1 complexes. The peroxidase-like activity was determined using the guaiacol, hydrogen peroxide system according to the method of Connell and Smithies ${ }^{3)}$ with some modifications. ${ }^{4)}$ The reaction mixture for the standard estimation procedure consisted of (1) $5 \mathrm{ml}$ of $0.03 \mathrm{M}$ guaiacol used as electron donor in $0.1 \mathrm{M}$ acetic acid adjusted to $\mathrm{pH} 4.0$ with $1 \mathrm{M}$ sodium hydroxide, (2) $0.5 \mathrm{ml}$ of the intermediate or saturated form ( $5 \mathrm{mg} / \mathrm{dl} \mathrm{Hb}$ concentration) converted to Met-Hb form with potassium ferricyanide, and (3) $1.0 \mathrm{ml}$ of $0.05 \mathrm{M}$ hydrogen peroxide used as oxidizing substrate prepared immediately before use.

The oxygen-equilibria of Hb-Hp 1-1 complexes. The continuous oxygen-equilibrium curves were recorded at $20^{\circ} \mathrm{C}$ automatically according to the method of Imai et al. ${ }^{5)}$ The intermediate and saturated 
complexes in their oxygenated forms were dialysed against $0.1 \mathrm{M}$ phosphate buffer $\mathrm{pH} 7.0$ for 24 hours and adjusted to 0.01 per cent $\mathrm{Hb}$ concentration with the same buffer used for dialysis. The oxygen pressure in the samples was determined with a Beckman 39065 polarographic oxygen sensor and the per cent saturation of $\mathrm{Hb}$ with oxygen was measured spectrophotometrically at $430 \mathrm{~m} \mu$

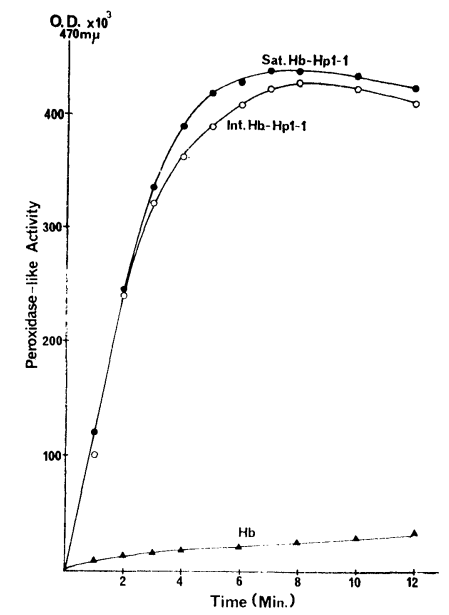

Fig. 1. Time plot of the peroxidase-like activity of $\mathrm{Hb}-\mathrm{Hp} 1-1$ complexes and free $\mathrm{Hb}$ by the standard estimation procedure.

Results. The peroxidase-like activity of Hb-Hp 1-1 complexes. Fig. 1 gives a time plot of the peroxidase-like activity of the intermediate and saturated forms obtained by the standard estimation procedure. The curves of the intermediate and saturated form showed rapid and linear increases during the initial 3 minutes and reached their maxima within 7 minutes. The initial reaction rate and the maximum color development of these two complexes were approximately 10 and 20 -fold higher than those of free $\mathrm{Hb}$ at identical heme concentration, but no significant difference was disclosed in the time course of the intermediate and saturated forms.

As shown in Fig. 2, pH of the reaction mixture was varied between 3.8 and 5.6. It will be noted that the optimum $\mathrm{pH}$ for free $\mathrm{Hb}$ is shifted to acidic in the presence of $\mathrm{Hp}$, and $\mathrm{pH}$ optimum for the initial reaction rate and the maximum color development were 3.8 and 4.6, respectively, either for the intermediate form or for the saturated form.

The effect of hydrogen peroxide concentration on the peroxidaselike activities of Hb-Hp 1-1 complexes is given in Fig. 3. The initial reaction rate of both of the intermediate and saturated forms in- 

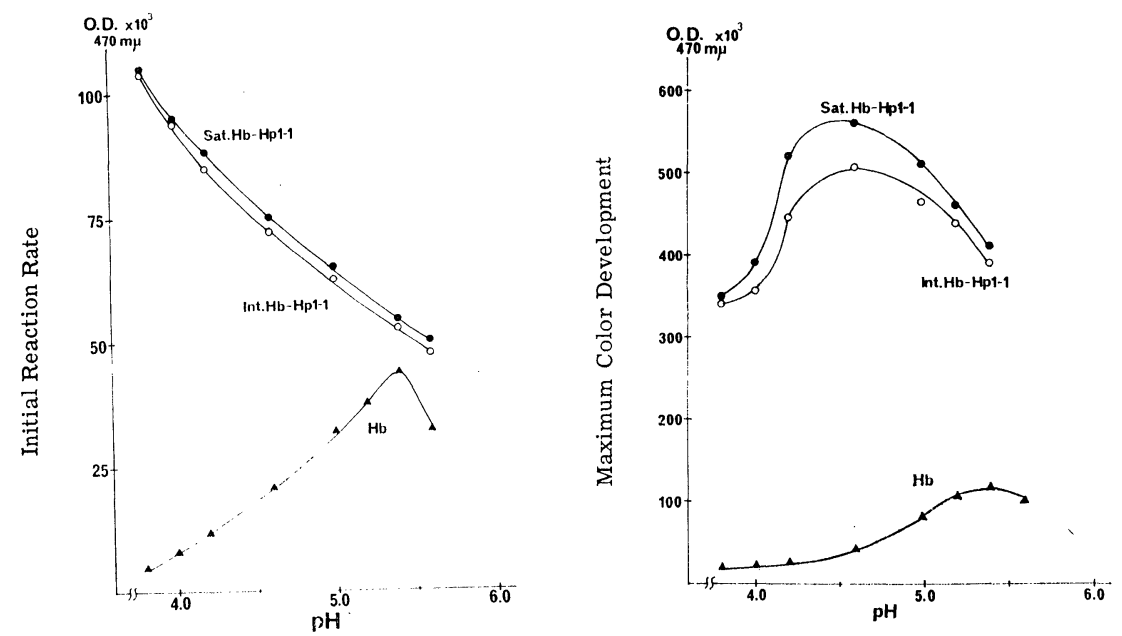

Fig. 2. Effect of $\mathrm{pH}$ on the peroxidase-like activity of $\mathrm{Hb}-\mathrm{Hp}$ 1-1 complexes and free $\mathrm{Hb}$.

creased in a similar fashion with increasing concentration of hydrogen peroxide between 12.5 and $400 \mathrm{mM}$, and the maximum color development of the two complexes was slightly decreased at the higher concentration of peroxide. Fig. 4 shows the influence of guaiacol concentration on the peroxidase-like activity of $\mathrm{Hb}-\mathrm{Hp}$ 1-1 complexes. The activity of the intermediate and saturated forms increased sharply up to $10 \mathrm{mM}$ and then decreased gradually either in the initial reaction rate or in the maximum color development. In contrast, the activity of free $\mathrm{Hb}$ decreased sharply with increasing concentration of guaiacol over $2.5 \mathrm{mM}$.

The oxygen-equilibria of $H b-H p 1-1$ complexes. The oxygen dissociation curves of $\mathrm{Hb}-\mathrm{Hp} 1-1$ complexes and free $\mathrm{Hb}$ were studied under identical conditions. The absorption spectra of these samples were checked between 350 and $650 \mathrm{~m} \mu$ before and after measurements using a Beckman DK-2 recording spectrophotometer. Methemoglobin formation during the measurement seemed neglisible, judging from changes in the absorption spectra. The absorption maxima for these two complexes, deoxygenated by addition of sodium dithionite after the measurements, were $428-430 \mathrm{~m} \mu$ in good agreement with that of free $\mathrm{Hb}$.

The oxygen-equilibrium curves of these two complexes were markedly different from that of free $\mathrm{Hb}$ which showed a typical sigmoidicity. Fig. 5 shows the oxygen dissociation curves plotted according to the logarithmic form of Hill's equation. Both of the intermediate and saturated forms had a high affinity for oxygen even at low oxygen pressure and the curves of these two complexes closely resemble each other. The value for $P_{1 / 2}$ observed in free 

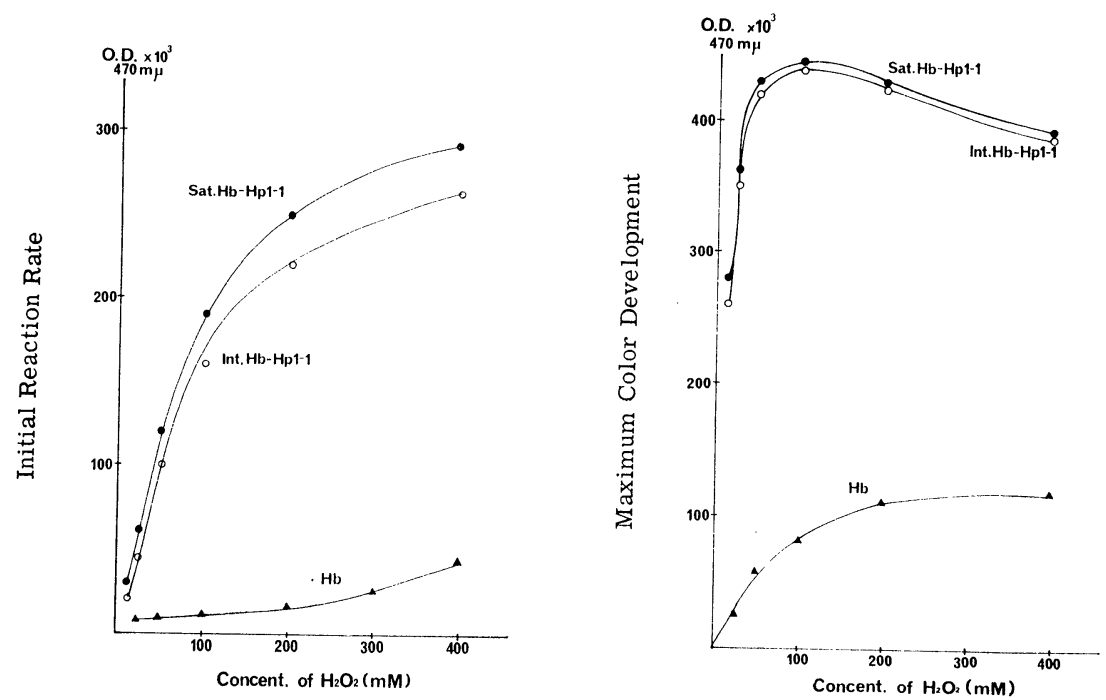

Fig. 3. Effect of concentration of hydrogen peroxide on the peroxidase-like activity of $\mathrm{Hb}-\mathrm{Hp}$ 1-1 complexes and free $\mathrm{Hb}$.
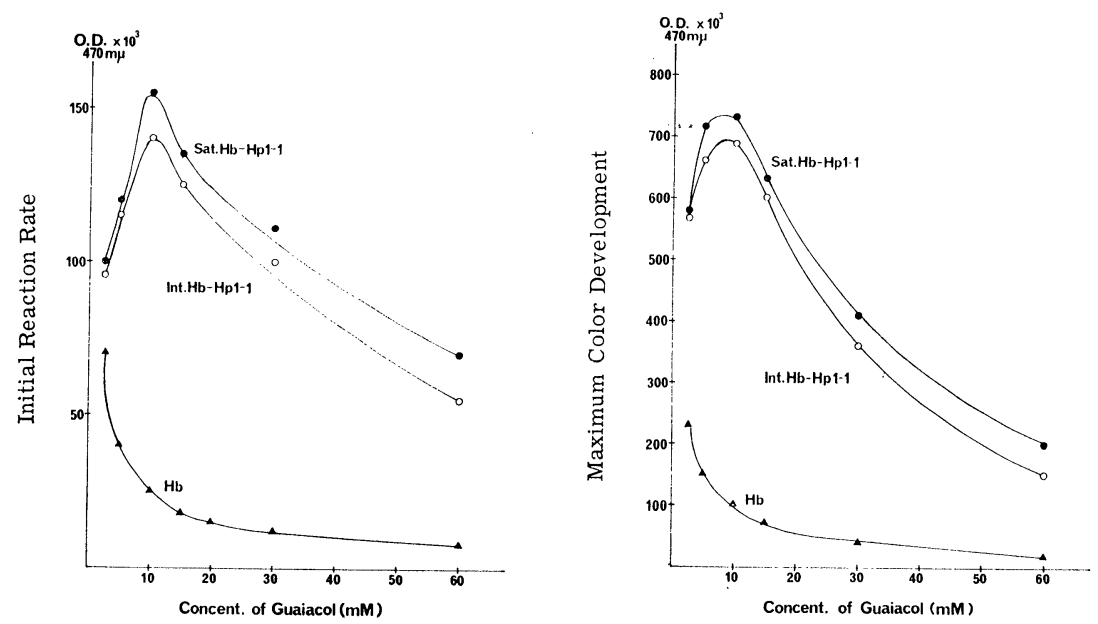

Fig. 4. Effect of concentration of guaiacol on the peroxidase-like activity of $\mathrm{Hb}-\mathrm{Hp} \mathrm{1-1}$ complexes and free $\mathrm{Hb}$.

$\mathrm{Hb}$ was $8.6 \mathrm{mmHg}$ and the value of $n$ was 2.8 . In contrast, the values of $\mathrm{P}_{1 / 2}$ were $1.2 \mathrm{mmHg}$ for the intermediate form and $1.4 \mathrm{mmHg}$ for the saturated form, and $n$ values of the intermediate and saturated forms were calculated to be 1.1 and 1.2, respectively.

Discussion. Recently Waks et al. ${ }^{6}{ }^{6}$ have proposed that the addition of an excess of human $\mathrm{Hp} \mathrm{1-1} \mathrm{to} \mathrm{horse} \mathrm{Hb}$ leads to the mixture of three components ; free $\mathrm{Hp}$, a complex of 1 mole of $\mathrm{Hp}$ and one $\mathrm{Hb}$ dimer and a complex of 1 mole of $\mathrm{Hp}$ and four $\mathrm{Hb}$ chains. These data, 


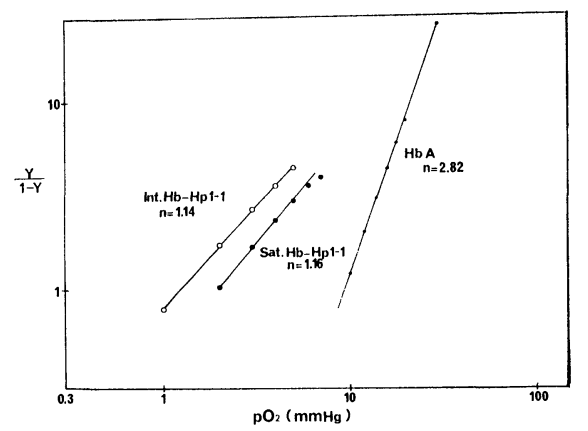

Fig. 5. Oxygen-equilibria of $\mathrm{Hb}-\mathrm{Hp}$ 1-1 complexes and free $\mathrm{Hb}$ at $\mathrm{pH} 7.0,20^{\circ} \mathrm{C}$, plotted according to the Hill's equation.

considered together, are consistent with our previous postulation that the intermediate and saturated forms would be Hp 1-1 bound of an $\alpha \beta$-subunit and two $\alpha \beta$-subunits of $\mathrm{Hb} \mathrm{A}$, and symmetrical splitting (into $\alpha \beta$-subunits) of $\mathrm{Hb} \mathrm{A}$ would occur by complex formation. ${ }^{1), 2)}$

The present studies showed no difference in the time course of the peroxidase-like activity of the intermediate and saturated forms. Concerning the activation mechanism of the peroxidase-like activity of $\mathrm{Hb}$, Waks and Alfsen ${ }^{7)}$ demonstrated that the maximum proton release during the association of $\mathrm{Hp}$ and $\mathrm{Hb}$, which parallels the rise in the peroxidase-like activity, occurs at $\mathrm{pH}$ 4.5. Connell and Smithies $^{3)}$ reported that $\mathrm{Hp}$ increases the peroxidase-like activity by protecting $\mathrm{Hb}$ from denaturation at low $\mathrm{pH}$ and peroxide. In the present studies, the optimum $\mathrm{pH}$ for both complexes was shifted to acidic $\mathrm{pH}$ and the effect of $\mathrm{pH}$ variation of the reaction mixture was almost identical in the intermediate and saturated forms. The inhibition of the peroxidase-like activity of $\mathrm{Hb}-\mathrm{Hp}$ complex and free $\mathrm{Hb}$ is said to be highly dependent on the concentration of hydrogen peroxide or guaiacol.3),4) Under the conditions studied here, the inhibition by various concentration of hydrogen peroxide or guaiacol was similar in these two complexes. These findings may be reasonably explained by the structural similarity of the Hb moiety within the intermediate and saturated forms.

Nagel et $a l .{ }^{8)}$ found that human $\mathrm{Hb}-\mathrm{Hp}$ 1-1 complex, corresponding to the saturated complex designated by the authors, has a high affinity for oxygen, the $n$ value of approximately 1 indicating absence of heme-heme interaction, and they ${ }^{9)}$ postulated that in $\mathrm{Hb}-\mathrm{Hp}$ 1-1 complex $\mathrm{Hb}$ can no longer undergo conformational change with ligand binding, and its structure is constrained in such a way that the heme groups can react only with a high ligand affinity.

The oxygen-equilibria of $\mathrm{Hb}-\mathrm{Hp} 1-1$ complexes obtained here 
indicate that the intermediate complex also has a high affinity for oxygen, the $n$ value of approximately 1 is almost identical with that of the saturated complex. These results may reflect the configurational changes of $\mathrm{Hb}$ moiety in these two complexes which make impossible the structural alteration underlying heme-heme interaction.

The functional changes, peroxidase-like activity and oxygen binding capacity, of the $\mathrm{Hb}$ moiety bound to $\mathrm{Hp}$ 1-1 demonstrated in the present studies are closely related to the configurational changes at heme-iron level induced by the binding of $\mathrm{Hb}$ to $\mathrm{Hp}$. Therefore, it will be pertinent to consider that these results seem to explain adequately the similarity in the structural changes occurring in the $\mathrm{Hb}$ moiety of the intermediate and saturated complexes.

Summary. The peroxidase-like activity of the intermediate and saturated forms of human hemoglobin-haptoglobin 1-1 complex was markedly higher than that of free hemoglobin, but no difference was seen in the activity of these two complexes under the various experimental conditions studied. Both the intermediate and saturated forms have a high affinity for oxygen with values of $n$ approximately 1 indicating the absence of heme-heme interaction.

From these findings and the data previously reported, it may be said that the configurational changes in the hemoglobin moiety induced by the association with haptoglobin 1-1 and thus the binding mechanism between haptoglobin 1-1 and hemoglobin closely related each other in the intermediate and saturated complexes.

Acknowledgement. This study was carried out as part of the JIBP and was suspported by a grant from the Ministry of Education. The authors are very grateful to Dr. H. B. Hamilton, Chief of Clinical Laboratories of ABCC in Hiroshima, for his careful reading of this paper.

\section{References}

1) Ogawa, A., Kagiyama, S., and Kawamura, K.: Proc. Japan Acad., 44, 1054 (1968).

2) Kagiyama, S., Ogawa, A., and Kawamura, K.: Proc. Japan Acad., 44, 1060 (1968).

3) Connell, G. E., and Smithies, O.: Biochem. J., 72, 115 (1959).

4) Mattenheimer, H., and Adams, E. C.: Z. klin. Chem. u. klin. Biochem., 2, 69 (1968).

5) Imai, K. et al.: Biochim. Biophys. Acta, 200, 189 (1970).

6) Waks, M. et al.: Archiv. Biochem. Biophys., 132, 268 (1969).

7) Waks, M., and Alfsen, A.: Biochim. Biophys. Res. Commun., 23, 62 (1966).

8) Nagel, R. N. et al.: Biochim. Biophys. Acta, 150, 286 (1965).

9) Nagel, R. N., and Gibson, Q. H.: J. Mol. Biol., 22, 249 (1966). 